\title{
MULTiPle SOLUTIONS FOR A NONLINEAR AND NON-HOMOGENEOUS PROBLEM IN ORLICZ-SOBOLEV SPACES
}

\author{
Mihai MihăILESCU ${ }^{a}$ AND DuŠAn Repovš ${ }^{b}$ \\ ${ }^{a}$ Department of Mathematics, Central European University, 1051 Budapest, Hungary \\ ${ }^{b}$ Faculty of Mathematics and Physics, and Faculty of Education, University of Ljubljana, \\ POB 2964, Ljubljana, Slovenia 1001 \\ E-mail addresses: mmihailes@yahoo.com dusan.repovs@guest.arnes.si
}

Abstract. We study a non-homogeneous boundary value problem in a smooth bounded domain in $\mathbb{R}^{N}$. We prove the existence of at least two nonnegative and non-trivial weak solutions. Our approach relies on Orlicz-Sobolev spaces theory combined with adequate variational methods and a variant of Mountain Pass Lemma.

2010 Mathematics Subject Classification: 35D30, 35J60, 35J70, 46N20, 58E05.

Key words: Non-homogeneous differential operator, Orlicz-Sobolev space, critical point, weak solution.

\section{Introduction and preliminary results}

Let $\Omega$ be a bounded domain in $\mathbb{R}^{N}(N \geq 3)$ with smooth boundary $\partial \Omega$. Assume that $a:(0, \infty) \rightarrow \mathbb{R}$ is a function such that the mapping $\varphi: \mathbb{R} \rightarrow \mathbb{R}$, defined by

$$
\varphi(t)= \begin{cases}a(|t|) t, & \text { for } t \neq 0 \\ 0, & \text { for } t=0\end{cases}
$$

is an odd, increasing homeomorphisms from $\mathbb{R}$ onto $\mathbb{R}$. This paper studies a nonlinear boundary value problem of the type

$$
\begin{cases}-\operatorname{div}(a(|\nabla u|) \nabla u)=\lambda f(x, u), & \text { for } \quad x \in \Omega \\ u=0, & \text { for } \quad x \in \partial \Omega\end{cases}
$$

where $f: \Omega \times \mathbb{R} \rightarrow \mathbb{R}$ is a Carathéodory function and $\lambda$ is a positive parameter.

In order to go further we introduce the functional space setting where problem (1) will be discussed. In this context we note that the operator in the divergence form is not homogeneous and thus, we introduce an Orlicz-Sobolev space setting for problems of this type.

The first general existence results using the theory of monotone operators in Orlicz-Sobolev spaces were obtained by Donaldson [6] and Gossez [8]. Other recent works that put the problem into this framework are contained in Clément et al. 4, 5], García-Huidobro et al. 7], Gossez and Manàsevich [9, Le and Schmitt [11, etc. In these papers, the existence results are obtained by means of variational techniques, monotone operator methods, or fixed point and degree theory arguments. The goal of our paper is to present a new multiplicity result for equations involving nonhomogeneous operators. Thus, it supplements the aforementioned results in the aspect that most of the papers guarantee existence but not multiplicity of solutions.

We start by recalling some basic facts about Orlicz spaces. For more details we refer to the books by D. R. Adams and L. L. Hedberg [1], R. Adams [2] and M. M. Rao and Z. D. Ren [17] and the papers by Ph. Clément et al. 4, 5], M. García-Huidobro et al. [7] and J. P. Gossez [8].

For $\varphi: \mathbb{R} \rightarrow \mathbb{R}$ introduced at the start of the paper, we define

$$
\Phi(t)=\int_{0}^{t} \varphi(s) d s, \quad \Phi^{\star}(t)=\int_{0}^{t} \varphi^{-1}(s) d s, \quad \text { for all } t \in \mathbb{R} .
$$


We observe that $\Phi$ is a Young function, that is, $\Phi(0)=0, \Phi$ is convex, and $\lim _{x \rightarrow \infty} \Phi(x)=+\infty$. Furthermore, since $\Phi(x)=0$ if and only if $x=0, \lim _{x \rightarrow 0} \Phi(x) / x=0$, and $\lim _{x \rightarrow \infty} \Phi(x) / x=+\infty$, then $\Phi$ is called an $N$-function. The function $\Phi^{\star}$ is called the complementary function of $\Phi$, and it satisfies

$$
\Phi^{\star}(t)=\sup \{s t-\Phi(s) ; s \geq 0\}, \quad \text { for all } t \geq 0 .
$$

We also observe that $\Phi^{\star}$ is also an $N$-function and the Young's inequality holds

$$
s t \leq \Phi(s)+\Phi^{\star}(t), \quad \text { for all } s, t \geq 0 .
$$

The Orlicz space $L_{\Phi}(\Omega)$ defined by the $N$-function $\Phi$ (see [1, 2, 4]) is the space of measurable functions $u: \Omega \rightarrow \mathbb{R}$ such that

$$
\|u\|_{L_{\Phi}}:=\sup \left\{\int_{\Omega} u v d x ; \int_{\Omega}(\Phi)^{\star}(|g|) d x \leq 1\right\}<\infty .
$$

Then $\left(L_{\Phi}(\Omega),\|\cdot\|_{L_{\Phi}}\right)$ is a Banach space whose norm is equivalent to the Luxemburg norm

$$
\|u\|_{\Phi}:=\inf \left\{k>0 ; \int_{\Omega} \Phi\left(\frac{u(x)}{k}\right) d x \leq 1\right\} .
$$

For Orlicz spaces Hölder's inequality reads as follows (see [17, Inequality 4, p. 79]):

$$
\int_{\Omega} u v d x \leq 2\|u\|_{L_{\Phi}}\|v\|_{L_{(\Phi) \star}} \quad \text { for all } u \in L_{\Phi}(\Omega) \text { and } v \in L_{(\Phi)^{\star}}(\Omega) .
$$

Next, we introduce the Orlicz-Sobolev spaces. We denote by $W^{1} L_{\Phi}(\Omega)$ the Orlicz-Sobolev space defined by

$$
W^{1} L_{\Phi}(\Omega):=\left\{u \in L_{\Phi}(\Omega) ; \frac{\partial u}{\partial x_{i}} \in L_{\Phi}(\Omega), i=1, \ldots, N\right\} .
$$

This is a Banach space with respect to the norm

$$
\|u\|_{1, \Phi}:=\|u\|_{\Phi}+\|\mid \nabla u\|_{\Phi} .
$$

We also define the Orlicz-Sobolev space $W_{0}^{1} L_{\Phi}(\Omega)$ as the closure of $C_{0}^{\infty}(\Omega)$ in $W^{1} L_{\Phi}(\Omega)$. By [8, Lemma $5.7]$ we may consider on $W_{0}^{1} L_{\Phi}(\Omega)$ the equivalent norm

$$
\|u\|:=\||\nabla u|\|_{\Phi} .
$$

For an easier manipulation of the spaces defined above, we define

$$
\varphi_{0}:=\inf _{t>0} \frac{t \varphi(t)}{\Phi(t)} \text { and } \varphi^{0}:=\sup _{t>0} \frac{t \varphi(t)}{\Phi(t)} .
$$

In this paper we assume that we have

$$
1<\varphi_{0} \leq \frac{t \varphi(t)}{\Phi(t)} \leq \varphi^{0}<\infty, \quad \forall t \geq 0 .
$$

The above relation implies that $\Phi$ satisfies the $\Delta_{2}$-condition, i.e.

$$
\Phi(2 t) \leq K \Phi(t), \quad \forall t \geq 0,
$$

where $K$ is a positive constant (see [15, Proposition 2.3]).

On the other hand, the following relations hold

$$
\|u\|^{\varphi^{0}} \leq \int_{\Omega} \Phi(|\nabla u|) d x \leq\|u\|^{\varphi_{0}}, \quad \forall u \in W_{0}^{1} L_{\Phi}(\Omega),\|u\|<1,
$$




$$
\|u\|^{\varphi_{0}} \leq \int_{\Omega} \Phi(|\nabla u|) d x \leq\|u\|^{\varphi^{0}}, \quad \forall u \in W_{0}^{1} L_{\Phi}(\Omega),\|u\|>1
$$

(see, e.g. [14, Lemma 1]).

Furthermore, in this paper we shall assume that the function $\Phi$ satisfies the following condition

$$
\text { the function }[0, \infty) \ni t \rightarrow \Phi(\sqrt{t}) \text { is convex . }
$$

Conditions (3) and (6) assure that the Orlicz space $L_{\Phi}(\Omega)$ is a uniformly convex space and thus, a reflexive Banach space (see [15, Proposition 2.2]). This fact implies that also the Orlicz-Sobolev space $W_{0}^{1} L_{\Phi}(\Omega)$ is a reflexive Banach space.

Remark. We point out certain examples of functions $\varphi: \mathbb{R} \rightarrow \mathbb{R}$ which are odd, increasing homeomorphisms from $\mathbb{R}$ onto $\mathbb{R}$ and satisfy conditions (2) and (6). For more details the reader can consult [5, Examples 1-3, p. 243].

1) Let

$$
\varphi(t)=p|t|^{p-2} t, \quad \forall t \in \mathbb{R},
$$

with $p>1$. For this function it can be proved that

$$
\varphi_{0}=\varphi^{0}=p
$$

Furthermore, in this particular case the corresponding Orlicz space $L_{\Phi}(\Omega)$ is the classical Lebesgue space $L^{p}(\Omega)$ while the Orlicz-Sobolev space $W_{0}^{1} L_{\Phi}(\Omega)$ is the classical Sobolev space $W_{0}^{1, p}(\Omega)$. We will use the classical notations to denote the Orlicz-Sobolev spaces in this particular case.

2) Consider

$$
\varphi(t)=\log \left(1+|t|^{s}\right)|t|^{p-2} t, \quad \forall t \in \mathbb{R},
$$

with $p, s>1$. In this case it can be proved that

$$
\varphi_{0}=p, \quad \varphi^{0}=p+s .
$$

3) Let

$$
\varphi(t)=\frac{|t|^{p-2} t}{\log (1+|t|)}, \quad \text { if } t \neq 0, \quad \varphi(0)=0,
$$

with $p>2$. In this case we have

$$
\varphi_{0}=p-1, \quad \varphi^{0}=p
$$

\section{The main result}

In this paper we study problem (11) in the special case when

$$
f(x, t)=t^{p-1}-t^{q-1}
$$

with

$$
1<q<p<\varphi_{0}
$$

and $t \geq 0$.

More precisely, we consider the degenerate boundary value problem

$$
\begin{cases}-\operatorname{div}(a(|\nabla u|) \nabla u)=\lambda\left(u^{p-1}-u^{q-1}\right), & \text { for } x \in \Omega \\ u=0, & \text { for } x \in \partial \Omega \\ u \geq 0, & \text { for } x \in \Omega .\end{cases}
$$


We say that $u \in W_{0}^{1} L_{\Phi}(\Omega)$ is a weak solution of problem (8) if $u \geq 0$ a. e. in $\Omega$ and

$$
\int_{\Omega} a(|\nabla u|) \nabla u \cdot \nabla v d x-\lambda \int_{\Omega} u^{p-1} v d x+\lambda \int_{\Omega} u^{q-1} v d x=0
$$

for all $v \in W_{0}^{1} L_{\Phi}(\Omega)$.

Our main result asserts that problem (8) has at least two nontrivial weak solutions provided that $\lambda>0$ is large enough. More precisely, we prove

Theorem 1. Assume that condition (7) is fulfilled and

$$
\varphi^{0}<\min \left\{N, \frac{N \varphi_{0}}{N-\varphi_{0}}\right\} .
$$

Then there exists $\lambda^{\star}>0$ such that for all $\lambda>\lambda^{\star}$ problem (8) has at least two distinct non-negative, nontrivial weak solutions.

Remark. We point out that our result was inspired by [16. Theorem 1.2], where a related property was proved in the case of the $p$-Laplace operators. The extension from $p$-Laplace operator to the differential operators involved in (8) is not trivial, since the new operators have a more complicated structure than the $p$-Laplace operator, for example they are non-homogeneous.

Finally, we mention that a similar study regarding the existence and multiplicity of solutions for equations involving the $p(x)$-Laplace operator can be found in Mihăilescu and Rădulescu [12.

\section{Proof of Theorem 1}

Let $E$ denote the generalized Sobolev space $W_{0}^{1} L_{\Phi}(\Omega)$.

Define the energy functional $I: E \rightarrow \mathbb{R}$ by

$$
I(u)=\int_{\Omega} \Phi(|\nabla u|) d x-\frac{\lambda}{\gamma} \int_{\Omega} u_{+}^{p} d x+\frac{\lambda}{\beta} \int_{\Omega} u_{+}^{q} d x,
$$

where $u_{+}(x)=\max \{u(x), 0\}$.

We remember that $u \in E$ implies $u_{+}, u_{-} \in E$ and

$$
\nabla u_{+}=\left\{\begin{array}{lll}
0, & \text { if } & {[u \leq 0]} \\
\nabla u, & \text { if } & {[u>0],}
\end{array} \quad \nabla u_{-}=\left\{\begin{array}{lll}
0, & \text { if } & {[u \geq 0]} \\
\nabla u, & \text { if } & {[u<0]}
\end{array}\right.\right.
$$

where $u_{ \pm}=\max \{ \pm u(x), 0\}$ for all $x \in \Omega$ (see, e.g. page 52 in [4]). That fact and some standards arguments assure that functional $I$ is well-defined on $E$ and $I \in C^{1}(E, \mathbb{R})$ with the derivative given by

$$
\left\langle I^{\prime}(u), v\right\rangle=\int_{\Omega} a(|\nabla u|) \nabla u \cdot \nabla v d x-\lambda \int_{\Omega} u_{+}^{p-1} v d x+\lambda \int_{\Omega} u_{+}^{q-1} v d x,
$$

for all $u, v \in E$.

Remark. We point out that if $u$ is a critical point of $I$ then using the above information and condition (2) we have

$$
\begin{aligned}
0 & =\left\langle I^{\prime}(u), u_{-}\right\rangle=\int_{\Omega} a(|\nabla u|) \nabla u \cdot \nabla u_{-} d x-\lambda \int_{\Omega}\left(u_{+}\right)^{p-1} u_{-} d x+\lambda \int_{\Omega}\left(u_{+}\right)^{q-1} u_{-} d x \\
& \left.=\int_{\Omega} a|\nabla u|\right) \nabla u \cdot \nabla u_{-} d x=\int_{\Omega} a\left(\left|\nabla u_{-}\right|\right)\left|\nabla u_{-}\right|^{2} d x \geq \varphi_{0} \int_{\Omega} \Phi\left(\left|\nabla u_{-}\right|\right) d x .
\end{aligned}
$$

By the above estimates and relation (4) we deduce that $u \geq 0$. It follows that the nontrivial critical points of $I$ are non-negative solutions of (8).

The above remark shows that we can prove Theorem 1 using the critical point theory. More exactly, we first show that for $\lambda>0$ large enough, the functional $I$ has a global minimizer $u_{1} \geq 0$ such that $I\left(u_{1}\right)<0$. Next, by means of the Mountain Pass Theorem, a second critical point $u_{2}$ with $I\left(u_{2}\right)>0$ is obtained. 
Lemma 1. There exists $\lambda_{1}>0$ such that

$$
\lambda_{1}=\inf _{u \in E,\|u\|>1} \frac{\int_{\Omega} \Phi(|\nabla u|) d x}{\int_{\Omega}|u|^{\varphi_{0}} d x} .
$$

Proof. First, we note that by condition (2) we can deduce that $E$ is continuously embedded in the classical Sobolev space $W_{0}^{1, \varphi_{0}}(\Omega)$. Consequently, $E$ is continuously embedded in the classical Lebesgue space $L^{\varphi_{0}}(\Omega)$. It follows that there exists $C>0$ such that

$$
\|u\| \geq C\|u\|_{L^{\varphi_{0}(\Omega)}}, \quad \forall u \in E .
$$

On the other hand, by (5) we have

$$
\int_{\Omega} \Phi(|\nabla u|) d x \geq\|u\|^{\varphi_{0}}, \quad \forall u \in E \text { with }\|u\|>1 .
$$

Combining the above inequalities we obtain

$$
\int_{\Omega} \Phi(|\nabla u|) d x \geq C^{\varphi_{0}} \int_{\Omega}|u|^{\varphi_{0}} d x, \quad \forall u \in E \text { with }\|u\|>1 .
$$

The proof of Lemma 1 is complete.

Proposition 1. (i) The functional I is bounded from below and coercive.

(ii) The functional I is weakly lower semi-continuous.

Proof. (i) Since $1<q<p<\varphi_{0}$ we have

$$
\lim _{t \rightarrow \infty} \frac{\frac{1}{p} t^{p}-\frac{1}{q} t^{q}}{t^{\varphi_{0}}}=0 .
$$

Then for any $\lambda>0$ there exists $C_{\lambda}>0$ such that

$$
\lambda\left(\frac{1}{p} t^{p}-\frac{1}{q} t^{q}\right) \leq \frac{\lambda_{1}}{2} t^{\varphi_{0}}+C_{\lambda}, \quad \forall t \geq 0,
$$

where $\lambda_{1}$ was defined in Lemma 1 .

The above inequality and condition (5) show that for any $u \in E$ with $\|u\|>1$ we have

$$
\begin{aligned}
I(u) & \geq \int_{\Omega} \Phi(|\nabla u|) d x-\frac{\lambda_{1}}{2} \int_{\Omega}|u|^{\varphi_{0}} d x-C_{\lambda} \mu(\Omega) \\
& \geq \frac{1}{2} \int_{\Omega} \Phi(|\nabla u|) d x-C_{\lambda} \mu(\Omega) \\
& \geq \frac{1}{2}\|u\|^{\varphi_{0}}-C_{\lambda} \mu(\Omega) .
\end{aligned}
$$

This shows that $I$ is bounded from below and coercive.

(ii) Similar arguments as those used in the proof of [13, Theorem 2] (see also [15, Lemma 4.3]) show that the functional $I_{0}: E \rightarrow \mathbb{R}$ defined by

$$
I_{0}(u)=\int_{\Omega} \Phi(|\nabla u|) d x
$$


is weakly lower semi-continuous. We justify that $I$ is weakly lower semi-continuous. Let $\left(u_{n}\right) \subset E$ be a sequence which converges weakly to $u$ in $E$. Since $I_{0}$ is weakly lower semi-continuous we have

$$
I_{0}(u) \leq \liminf _{n \rightarrow \infty} I_{0}\left(u_{n}\right) .
$$

On the other hand, since $E$ is compactly embedded in $L^{p}(\Omega)$ and $L^{q}(\Omega)$ it follows that $\left(u_{n_{+}}\right)$converges strongly to $u_{+}$both in $L^{p}(\Omega)$ and in $L^{q}(\Omega)$. (The compact embedding of $E$ into $L^{p}(\Omega)$ and $L^{q}(\Omega)$ is a direct consequence of the fact that $E$ is continuously embedded in the classical Sobolev space $W_{0}^{1, \varphi_{0}}(\Omega)$ combined with condition (7).) This fact together with relation (111) imply

$$
I(u) \leq \liminf _{n \rightarrow \infty} I\left(u_{n}\right) .
$$

Therefore, $I$ is weakly lower semi-continuous. The proof of Proposition 1 is complete.

From Proposition 1 and Theorem 1.2 in 18 we deduce that there exists $u_{1} \in E$ a global minimizer of $I$. The following result implies that $u_{1} \neq 0$, provided that $\lambda$ is sufficiently large.

Proposition 2. There exists $\lambda^{\star}>0$ such that $\inf _{E} I<0$.

Proof. Let $\Omega_{1} \subset \Omega$ be a compact subset, large enough and $u_{0} \in E$ be such that $u_{0}(x)=t_{0}$ in $\Omega_{1}$ and $0 \leq u_{0}(x) \leq t_{0}$ in $\Omega \backslash \Omega_{1}$, where $t_{0}>1$ is chosen such that

$$
\frac{1}{p} t_{0}^{p}-\frac{1}{q} t_{0}^{q}>0 \text {. }
$$

We have

$$
\begin{aligned}
\frac{1}{p} \int_{\Omega} u_{0}^{p} d x-\frac{1}{q} \int_{\Omega} u_{0}^{q} d x & \geq \frac{1}{p} \int_{\Omega_{1}} u_{0}^{p} d x-\frac{1}{q} \int_{\Omega_{1}} u_{0}^{q} d x-\frac{1}{q} \int_{\Omega_{\Omega}} u_{0}^{q} d x \\
& \geq \frac{1}{p} \int_{\Omega_{1}} u_{0}^{p} d x-\frac{1}{q} \int_{\Omega_{1}} u_{0}^{q} d x-\frac{1}{q} t_{0}^{q} \mu\left(\Omega \backslash \Omega_{1}\right)>0
\end{aligned}
$$

and thus $I\left(u_{0}\right)<0$ for $\lambda>0$ large enough. The proof of Proposition 2 is complete.

Since Proposition 2 holds it follows that $u_{1} \in E$ is a nontrivial weak solution of problem (8).

Fix $\lambda \geq \lambda^{\star}$. Set

$$
g(x, t)= \begin{cases}0, & \text { for } t<0 \\ t^{p-1}-t^{q-1}, & \text { for } 0 \leq t \leq u_{1}(x) \\ u_{1}(x)^{p-1}-u_{1}(x)^{q-1}, & \text { for } t>u_{1}(x)\end{cases}
$$

and

$$
G(x, t)=\int_{0}^{t} g(x, s) d s .
$$

Define the functional $J: E \rightarrow \mathbb{R}$ by

$$
J(u)=\int_{\Omega} \Phi(|\nabla u|) d x-\lambda \int_{\Omega} G(x, u) d x .
$$

The same arguments as those used for functional $I$ imply that $J \in C^{1}(E, \mathbb{R})$ and

$$
\left\langle J^{\prime}(u), v\right\rangle=\int_{\Omega} a(|\nabla u|) \nabla u \cdot \nabla v d x-\lambda \int_{\Omega} g(x, u) v d x,
$$

for all $u, v \in E$.

On the other hand, we point out that if $u \in E$ is a critical point of $J$ then $u \geq 0$. The proof can be carried out as in the case of functional $I$.

Next, we prove 
Lemma 2. If $u$ is a critical point of $J$ then $u \leq u_{1}$.

Proof. We have

$$
\begin{aligned}
0 & =\left\langle J^{\prime}(u)-I^{\prime}\left(u_{1}\right),\left(u-u_{1}\right)_{+}\right\rangle \\
& =\int_{\Omega}\left(a(|\nabla u|) \nabla u-a\left(\left|\nabla u_{1}\right|\right) \nabla u_{1}\right) \cdot \nabla\left(u-u_{1}\right)_{+} d x-\lambda \int_{\Omega}\left[g(x, u)-\left(u_{1}^{p-1}-u_{1}^{q-1}\right)\right]\left(u-u_{1}\right)_{+} d x \\
& =\int_{\left[u>u_{1}\right]}\left(a(|\nabla u|) \nabla u-a\left(\left|\nabla u_{1}\right|\right) \nabla u_{1}\right) \cdot \nabla\left(u-u_{1}\right) d x .
\end{aligned}
$$

Notice that since $\varphi$ is increasing in $\mathbb{R}$ we have for each $\xi$ and $\psi \in \mathbb{R}^{N}$

$$
(\varphi(|\xi|)-\varphi(|\psi|))(|\xi|-|\psi|) \geq 0,
$$

with equality if and only if $\xi=\psi$. Thus, we can deduce that

$$
(a(|\xi|)|\xi|-a(|\psi|)|\psi|)(|\xi|-|\psi|) \geq 0,
$$

for all $\xi, \psi \in \mathbb{R}^{N}$, with equality if and only if $\xi=\psi$. On the other hand, some simple computations show that

$$
(a(|\xi|) \xi-a(|\psi|) \psi) \cdot(\xi-\psi) \geq(a(|\xi|)|\xi|-\varphi(|\psi|)|\psi|)(|\xi|-|\psi|),
$$

for all $\xi, \psi \in \mathbb{R}^{N}$. Consequently, we conclude that

$$
(a(|\xi|) \xi-a(|\psi|) \psi) \cdot(\xi-\psi) \geq 0,
$$

for all $\xi, \psi \in \mathbb{R}^{N}$, with equality if and only if $\xi=\psi$.

Using the above pieces of information we deduce that the above equality holds if and only if $\nabla u=\nabla u_{1}$. It follows that $\nabla u(x)=\nabla u_{1}(x)$ for all $x \in \omega:=\left\{y \in \Omega ; u(y)>u_{1}(y)\right\}$. Hence

$$
\int_{\omega} \Phi\left(\left|\nabla\left(u-u_{1}\right)\right|\right) d x=0
$$

and thus

$$
\int_{\Omega} \Phi\left(\left|\nabla\left(u-u_{1}\right)_{+}\right|\right) d x=0
$$

By relation (4) we obtain

$$
\left\|\left(u-u_{1}\right)_{+}\right\|=0 .
$$

We obtain that $\left(u-u_{1}\right)_{+}=0$ in $\Omega$, that is, $u \leq u_{1}$ in $\Omega$. The proof of Lemma 2 is complete.

In the following we determine a critical point $u_{2} \in E$ of $J$ such that $J\left(u_{2}\right)>0$ via the Mountain Pass Theorem. By the above lemma we will deduce that $0 \leq u_{2} \leq u_{1}$ in $\Omega$. Therefore

$$
g\left(x, u_{2}\right)=u_{2}^{p-1}-u_{2}^{q-1} \text { and } G\left(x, u_{2}\right)=\frac{1}{p} u_{2}^{p}-\frac{1}{q} u_{2}^{q}
$$

and thus

$$
J\left(u_{2}\right)=I\left(u_{2}\right) \quad \text { and } \quad J^{\prime}\left(u_{2}\right)=I^{\prime}\left(u_{2}\right)
$$

More precisely, we find

$$
I\left(u_{2}\right)>0=I(0)>I\left(u_{1}\right) \quad \text { and } \quad I^{\prime}\left(u_{2}\right)=0 .
$$

This shows that $u_{2}$ is a weak solution of problem (8) such that $0 \leq u_{2} \leq u_{1}, u_{2} \neq 0$ and $u_{2} \neq u_{1}$.

In order to find $u_{2}$ described above we prove

Lemma 3. There exists $\rho \in\left(0,\left\|u_{1}\right\|\right)$ and $a>0$ such that $J(u) \geq a$, for all $u \in E$ with $\|u\|=\rho$. 
Proof. Let $u \in E$ be fixed, such that $\|u\|<1$. It is clear that there exists $\delta>1$ such that

$$
\frac{1}{p} t^{p}-\frac{1}{q} t^{q} \leq 0, \quad \forall t \in[0, \delta] .
$$

For $\delta$ given above we define

$$
\Omega_{u}=\{x \in \Omega ; u(x)>\delta\} .
$$

If $x \in \Omega \backslash \Omega_{u}$ with $u(x)<u_{1}(x)$ we have

$$
G(x, u)=\frac{1}{p} u_{+}^{p}-\frac{1}{q} u_{+}^{q} \leq 0 .
$$

If $x \in \Omega \backslash \Omega_{u}$ with $u(x)>u_{1}(x)$ then $u_{1}(x) \leq \delta$ and we have

$$
G(x, u)=\frac{1}{p} u_{1}^{p}-\frac{1}{q} u_{1}^{q} \leq 0 .
$$

Thus we deduce that

$$
G(x, u) \leq 0, \quad \text { on } \Omega \backslash \Omega_{u} .
$$

Provided that $\|u\|<1$ by relation (4) we get

$$
\begin{aligned}
J(u) & \geq \int_{\Omega} \Phi(|\nabla u|) d x-\lambda \int_{\Omega_{u}} G(x, u) d x \\
& \geq\|u\|^{\varphi^{0}}-\lambda \int_{\Omega_{u}} G(x, u) d x
\end{aligned}
$$

By relation (9) it follows that $\varphi^{0}<\varphi_{0}^{\star}:=\frac{N \varphi_{0}}{N-\varphi_{0}}$. On the other hand, as we already pointed out, by condition (2) we deduce that $E$ is continuously embedded in the classical Sobolev space $W_{0}^{1, \varphi_{0}}(\Omega)$. Consequently, there exists $s \in\left(\varphi^{0}, \frac{N \varphi_{0}}{N-\varphi_{0}}\right)$ such that $E$ is continuously embedded in the classical Lebesgue space $L^{s}(\Omega)$. Thus, there exists a positive constant $C>0$ such that

$$
\|u\|_{L^{s}(\Omega)} \leq C\|u\|, \quad \forall u \in E .
$$

Using the definition of $G$, Hölder's inequality and the above estimate, we obtain

$$
\begin{aligned}
\lambda \int_{\Omega_{u}} G(x, u) d x & =\lambda \int_{\Omega_{u} \cap\left[u<u_{1}\right]}\left(\frac{1}{p} u_{+}^{p}-\frac{1}{q} u_{+}^{q}\right) d x+\lambda \int_{\Omega_{u} \cap\left[u>u_{1}\right]}\left(\frac{1}{p} u_{1}^{p}-\frac{1}{q} u_{1}^{q}\right) d x \\
& \leq \frac{2 \lambda}{p} \int_{\Omega_{u}} u_{+}^{p} d x \\
& \leq \frac{2 \lambda}{p} \int_{\Omega_{u}} u_{+}^{\varphi^{0}} d x \\
& \leq \frac{2 \lambda}{p}\left(\int_{\Omega_{u}} u_{+}^{s} d x\right)^{\varphi^{0} / s}\left[\mu\left(\Omega_{u}\right)\right]^{1-\varphi^{0} / q} \\
& \leq C \frac{2 \lambda}{p}\left[\mu\left(\Omega_{u}\right)\right]^{1-\varphi^{0} / s}\|u\|^{\varphi^{0}}
\end{aligned}
$$

By (12) and (13) we infer that it is enough to show that $\mu\left(\Omega_{u}\right) \rightarrow 0$ as $\|u\| \rightarrow 0$ in order to prove Lemma 3

Let $\epsilon>0$. We choose $\Omega_{\epsilon} \subset \Omega$ a compact subset, such that $\mu\left(\Omega \backslash \Omega_{\epsilon}\right)<\epsilon$. We denote by $\Omega_{u, \epsilon}:=\Omega_{u} \cap \Omega_{\epsilon}$. Then it is clear that

$$
C^{\varphi^{0}}\|u\|^{\varphi^{0}} \geq\left(\int_{\Omega}|u|^{s} d x\right)^{\varphi^{0} / s} \geq\left(\int_{\Omega_{u, \epsilon}}|u|^{s} d x\right)^{\varphi^{0} / s} \geq \delta^{\varphi^{0}}\left[\mu\left(\Omega_{u, \epsilon}\right)\right]^{\varphi^{0} / s} .
$$


The above inequality implies that $\mu\left(\Omega_{u, \epsilon}\right) \rightarrow 0$ as $\|u\| \rightarrow 0$.

Since $\Omega_{u} \subset \Omega_{u, \epsilon} \cup\left(\Omega \backslash \Omega_{\epsilon}\right)$ we have

$$
\mu\left(\Omega_{u}\right) \leq \mu\left(\Omega_{u, \epsilon}\right)+\epsilon
$$

and $\epsilon>0$ is arbitrary. We find that $\mu\left(\Omega_{u}\right) \rightarrow 0$ as $\|u\| \rightarrow 0$. This concludes the proof of Lemma 3 .

Lemma 4. The functional $J$ is coercive.

Proof. For each $u \in E$ with $\|u\|>1$ by relation (5D) and Hölder's inequality we have

$$
\begin{aligned}
J(u) & \geq \int_{\Omega} \Phi(|\nabla u|) d x-\lambda \int_{\left[u>u_{1}\right]} G(x, u) d x-\lambda \int_{\left[u<u_{1}\right]} G(x, u) d x \\
& \geq\|u\|^{\varphi_{0}}-\frac{\lambda}{p} \int_{\left[u>u_{1}\right]} u_{1}^{p} d x+\frac{\lambda}{q} \int_{\left[u>u_{1}\right]} u_{1}^{q} d x-\frac{\lambda}{p} \int_{\left[u<u_{1}\right]} u_{+}^{p} d x+\frac{\lambda}{q} \int_{\left[u<u_{1}\right]} u_{+}^{q} d x \\
& \geq\|u\|^{\varphi_{0}}-\frac{\lambda}{p} \int_{\Omega} u_{1}^{p} d x-\frac{\lambda}{p} \int_{\Omega} u_{+}^{p} d x \\
& \geq\|u\|^{\varphi_{0}}-\frac{\lambda}{p}[\mu(\Omega)]^{1-p / \varphi_{0}} C_{1}\|u\|^{p}-C_{2} \\
& \geq\|u\|^{\varphi_{0}}-C_{2} 3\|u\|^{p}-C_{2},
\end{aligned}
$$

where $C_{1}, C_{2}$ and $C_{3}$ are positive constants. Since $p<\varphi_{0}$ the above inequality implies that $J(u) \rightarrow \infty$ as $\|u\| \rightarrow \infty$, that is, $J$ is coercive. The proof of Lemma 4 is complete.

The following result yields a sufficient condition which ensures that a weakly convergent sequence in $E$ converges strongly, too.

Lemma 5. Assume that the sequence $\left(u_{n}\right)$ converges weakly to $u$ in $E$ and

$$
\limsup _{n \rightarrow \infty} \int_{\Omega} a\left(\left|\nabla u_{n}\right|\right) \nabla u_{n} \cdot\left(\nabla u_{n}-\nabla u\right) d x \leq 0 .
$$

Then $\left(u_{n}\right)$ converges strongly to $u$ in $E$.

Proof. Since $u_{n}$ converges weakly to $u$ in $E$ implies that it follows that $\left(\left\|u_{n}\right\|\right)$ is a bounded sequence of real numbers. That fact and relations (44) and (5) imply thet the sequence $\left(I_{0}\left(u_{n}\right)\right)$ is bounded, where $I_{0}$ is defined by relation (10). Then, up to to a subsequence, we deduce that $I_{0}\left(u_{n}\right) \rightarrow c$. Furthermore, the weak lower semi-continuity of $I_{0}$ (pointed out above) implies

$$
I_{0}(u) \leq \liminf _{n \rightarrow \infty} I_{0}\left(u_{n}\right)=c .
$$

On the other hand, since $I_{0}$ is convex (because $\Phi$ is convex), we have

$$
I_{0}(u) \geq I_{0}\left(u_{n}\right)+\left\langle I_{0}^{\prime}\left(u_{n}\right), u-u_{n}\right\rangle .
$$

Next, by the hypothesis $\lim \sup \int_{\Omega} a\left(\left|\nabla u_{n}\right|\right) \nabla u_{n} \cdot\left(\nabla u_{n}-\nabla u\right) d x \leq 0$, we conclude that $I_{0}(u)=c$.

Taking into account that $\left(u_{n}+u\right) / 2$ converges weakly to $u$ in $E$ and using again the weak lower semi-continuity of $I_{0}$ we find

$$
c=I_{0}(u) \leq \liminf _{n \rightarrow \infty} I_{0}\left(\frac{u_{n}+u}{2}\right) .
$$

We assume by contradiction that $u_{n}$ does not converge to $u$ in $E$. Then by (4) it follows that there exist $\epsilon>0$ and a subsequence $\left(u_{n_{m}}\right)$ of $\left(u_{n}\right)$ such that

$$
I_{0}\left(\frac{u_{n_{m}}-u}{2}\right) \geq \epsilon, \quad \forall m
$$


On the other hand, relations (3) and (6) enable us to apply [10, Lemma 2.1] in order to obtain

$$
\frac{1}{2} I_{0}(u)+\frac{1}{2} I_{0}\left(u_{n_{m}}\right)-I_{0}\left(\frac{u+u_{n_{m}}}{2}\right) \geq I_{0}\left(\frac{u-u_{n_{m}}}{2}\right) \geq \epsilon, \quad \forall m .
$$

Letting $m \rightarrow \infty$ in the above inequality we obtain

$$
c-\epsilon \geq \limsup _{m \rightarrow \infty} I_{0}\left(\frac{u+u_{n_{m}}}{2}\right) d x,
$$

and that is a contradiction with (14). It follows that $u_{n}$ converges strongly to $u$ in $E$ and Lemma 5$]$ is proved.

Proof of Theorem 1 completed. Using Lemma 3 and the Mountain Pass Theorem (see [3] with the variant given by Theorem 1.15 in [19]) we deduce that there exists a sequence $\left(u_{n}\right) \subset E$ such that

$$
J\left(u_{n}\right) \rightarrow c>0 \text { and } \quad J^{\prime}\left(u_{n}\right) \rightarrow 0
$$

where

$$
c=\inf _{\gamma \in \Gamma} \max _{t \in[0,1]} J(\gamma(t))
$$

and

$$
\Gamma=\left\{\gamma \in C([0,1], E) ; \gamma(0)=0, \gamma(1)=u_{1}\right\} .
$$

By relation (17) and Lemma 4 we obtain that $\left(u_{n}\right)$ is bounded and thus passing eventually to a subsequence, still denoted by $\left(u_{n}\right)$, we may assume that there exists $u_{2} \in E$ such that $u_{n}$ converges weakly to $u_{2}$. Since $E$ is compactly embedded in $L^{i}(\Omega)$ for any $i \in\left[1, \varphi_{0}\right]$, it follows that $u_{n}$ converges strongly to $u_{2}$ in $L^{i}(\Omega)$ for all $i \in\left[1, \varphi_{0}\right]$. Hence

$$
\left\langle I_{0}^{\prime}\left(u_{n}\right)-I_{0}^{\prime}\left(u_{2}\right), u_{n}-u_{2}\right\rangle=\left\langle J^{\prime}\left(u_{n}\right)-J^{\prime}\left(u_{2}\right), u_{n}-u_{2}\right\rangle+\lambda \int_{\Omega}\left[g\left(x, u_{n}\right)-g\left(x, u_{2}\right)\right]\left(u_{n}-u_{2}\right) d x=o(1),
$$

as $n \rightarrow \infty$, where $I_{0}$ is defined by relation (10). By Lemma 5 we deduce that $u_{n}$ converges strongly to $u_{2}$ in $E$ and using relation (17) we find

$$
J\left(u_{2}\right)=c>0 \text { and } J^{\prime}\left(u_{2}\right)=0 .
$$

Therefore, $J\left(u_{2}\right)=c>0$ and $J^{\prime}\left(u_{2}\right)=0$. By Lemma 2 we deduce that $0 \leq u_{2} \leq u_{1}$ in $\Omega$. Therefore

$$
g\left(x, u_{2}\right)=u_{2}^{p-1}-u_{2}^{q-1} \text { and } G\left(x, u_{2}\right)=\frac{1}{p} u_{2}^{p}-\frac{1}{q} u_{2}^{q}
$$

and thus

$$
J\left(u_{2}\right)=I\left(u_{2}\right) \text { and } J^{\prime}\left(u_{2}\right)=I^{\prime}\left(u_{2}\right) .
$$

We conclude that $u_{2}$ is a critical point of $I$ and thus a solution of (8). Furthermore, $I\left(u_{2}\right)=c>0$ and $I\left(u_{2}\right)>0>I\left(u_{1}\right)$. Thus $u_{2}$ is not trivial and $u_{2} \neq u_{1}$. The proof of Theorem 1 is now complete.

Acknowledgements. This research was supported by Slovenian Research Agency grants P1-0292-0101 and J1-2057-0101.

\section{References}

[1] D. R. Adams and L. I. Hedberg, Function Spaces and Potential Theory, Grundlehren der Mathematischen Wissenschaften, 314, Springer-Verlag, Berlin, 1996.

[2] R. Adams, Sobolev Spaces, Academic Press, New York, 1975. 
[3] A. Ambrosetti and P. H. Rabinowitz, Dual variational methods in critical point theory, J. Funct. Anal. 14 (1973), 349-381.

[4] Ph. Clément, M. García-Huidobro, R. Manásevich, and K. Schmitt, Mountain pass type solutions for quasilinear elliptic equations, Calc. Var. 11 (2000), 33-62.

[5] Ph. Clément, B. de Pagter, G. Sweers, and F. de Thélin, Existence of solutions to a semilinear elliptic system through Orlicz-Sobolev spaces, Mediterr. J. Math. 1 (2004), 241-267.

[6] T. K. Donaldson, Nonlinear elliptic boundary value problems in Orlicz-Sobolev spaces, J. Differential Equations 10 (1971), 507-528

[7] M. García-Huidobro, V. K. Le, R. Manásevich, and K. Schmitt, On principal eigenvalues for quasilinear elliptic differential operators: an Orlicz-Sobolev space setting, Nonlinear Differential Equations Appl. (NoDEA) 6 (1999), 207-225.

[8] J. P. Gossez, Nonlinear elliptic boundary value problems for equations with rapidly (or slowly) increasing coefficients, Trans. Amer. Math. Soc. 190 (1974), 163-205.

[9] J.P. Gossez and R. Manàsevich, On a nonlinear eigenvalue problem in Orlicz-Sobolev spaces, Proc. Roy. Soc. Edinburgh Sect. A 132 (2002), 891-909.

[10] J. Lamperti, On the isometries of certain function-spaces, Pacific J. Math 8 (1958), 459-466.

[11] V. K. Le and K. Schmitt, Quasilinear elliptic equations and inequalities with rapidly growing coefficients, J. London Math. Soc. 62 (2000), 852-872.

[12] M. Mihăilescu and V. Rădulescu, A multiplicity result for a nonlinear degenerate problem arising in the theory of electrorheological fluids, Proceedings of the Royal Society A: Mathematical, Physical and Engineering Sciences 462 (2006), 2625-2641.

[13] M. Mihăilescu and V. Rădulescu, Existence and multiplicity of solutions for quasilinear nonhomogeneous problems: an Orlicz-Sobolev space setting, Journal of Mathematical Analysis and Applications 330 (2007), 416-432.

[14] M. Mihăilescu and V. Rădulescu, Eigenvalue problems associated to nonhomogeneous differential operators in OrliczSobolev spaces, Analysis and Applications 6 (2008), No. 1, 1-16.

[15] M. Mihăilescu and V. Rădulescu, Neumann problems associated to nonhomogeneous differential operators in OrliczSobolev spaces, Ann. Inst. Fourier 58 (6) (2008), 2087-2111.

[16] K. Perera, Multiple positive solutions for a class of quasilinear elliptic boundary-value problems, Electronic Journal of Differential Equations 7 (2003), 1-5.

[17] M. M. Rao and Z. D. Ren, Theory of Orlicz Spaces, Marcel Dekker, Inc., New York, 1991.

[18] M. Struwe, Variational Methods: Applications to Nonlinear Partial Differential Equations and Hamiltonian Systems, Springer, Heidelberg, 1996.

[19] M. Willem, Minimax Theorems, Birkhäuser, Boston, 1996. 